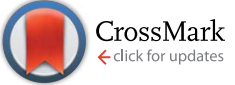

Cite this: RSC Adv., 2017, 7, 10376

Received 26th December 2016 Accepted 1st February 2017

DOI: $10.1039 / c 6 r a 28724 g$

rsc.li/rsc-advances

\title{
Self-assembly of porous $\mathrm{CuO}$ nanospheres decorated on reduced graphene oxide with enhanced lithium storage performance $\uparrow$
}

\author{
Gangyong Li, Mingjun Jing, ${ }^{\star}$ Zhengu Chen, Binhong He, Minjie Zhou \\ and Zhaohui Hou*
}

\begin{abstract}
This work aims at enhancing the cycling stability and rate capability of a CuO-based anode material for lithium-ion batteries. Here porous $\mathrm{CuO}$ nanospheres decorated on reduced graphene oxide (CuO-NSs/ $\mathrm{RGO}$ ) have been synthesized by a two-step thermal treatment procedure. The porous $\mathrm{CuO}$ nanospheres are assembled by ultra-fine nanoparticles of $\mathrm{CuO}$ with a size of $\sim 15 \mathrm{~nm}$. Such a porous nature endows many merits, improving the lithium storage performances of the CuO-NSs/RGO composite used as a lithium-ion battery anode. The porous CuO-NSs/RGO composite demonstrates superior reversible capacity $\left(753.3 \mathrm{~mA} \mathrm{~h} \mathrm{~g}^{-1}\right.$ at $\left.100 \mathrm{~mA} \mathrm{~g}^{-1}\right)$ and good cycling stability $\left(616.2 \mathrm{~mA} \mathrm{~h} \mathrm{~g}^{-1}\right.$ after 200 cycles at $500 \mathrm{~mA} \mathrm{~g}^{-1}$ ). In particular, it exhibits an outstanding high-rate capability of $327.3 \mathrm{~mA} \mathrm{~h} \mathrm{~g}^{-1}$ even at $5 \mathrm{~A}$ $\mathrm{g}^{-1}$. The feasibility of the CuO-NSs/RGO composite as an anode material was further investigated with a commercial $\mathrm{LiFePO}_{4}$ (LFP) cathode for lithium-ion batteries.
\end{abstract}

\section{Introduction}

Transition-metal oxides (TMOs) have great potential as anode candidates for high-performance lithium-ion batteries (LIBs) due to their large theoretical specific capacity based on the reversible electrochemical conversion reaction with lithium..$^{1-7}$ $\mathrm{CuO}$, as a member of the TMO family, has attracted significant attention because of its remarkably high theoretical capacities (672 $\mathrm{mA} \mathrm{h} \mathrm{g}^{-1}$ ), nontoxic nature, and eco-friendliness. ${ }^{8-10}$ However, given its intrinsic properties (e.g., inferior electrical conductivity, low lithium diffusivity, large volume expansion and contraction during lithiation/delithiation), $\mathrm{CuO}$ as anode electrode for LIBs has limitations, including large initial irreversible capacity loss, poor cycling stability, and undesirable rate performance. ${ }^{\mathbf{1 1 2}}$ To tackle these drawbacks and improve the electrochemical properties, designing novel electrode materials with advanced structures is critical for $\mathrm{CuO}$ anodes. To date, porous $\mathrm{CuO}$ nanorods, ${ }^{8}$ hierarchical $\mathrm{CuO}$ microspheres, ${ }^{13}$ urchin-like and shuttle-like $\mathrm{CuO},{ }^{\mathbf{1 4}}$ nanoplate-like $\mathrm{CuO},{ }^{15} \mathrm{CuO}$ nanowire, ${ }^{16}$ and leaf-like $\mathrm{CuO}^{17}$ have been synthesized and exhibit enhanced anodic performance due to their improved electrical/ionic conductivity and mechanical stability. In addition, another effective strategy is to incorporate

School of Chemistry and Chemical Engineering, Hunan Institute of Science and Technology, Yueyang 414006, China. E-mail: zhaohuihou@126.com; jingmingjun86@163.com

$\dagger$ Electronic supplementary information (ESI) available: Self-assembly of porous $\mathrm{CuO}$ nanospheres decorated on reduced graphene oxide with enhanced lithium storage performance. See DOI: $10.1039 / \mathrm{c} 6 \mathrm{ra} 28724 \mathrm{~g}$ carbonaceous material into the nanocomposites, such as pyrolysis carbon, ${ }^{11}$ carbon nanotubes, ${ }^{18-21}$ and graphene. ${ }^{12,22-24}$ Among these carbonaceous materials, graphene as a one-atomthick layer of carbon is now becoming one of the most attractive matrices owing to its superior electrical, mechanical and chemical properties. ${ }^{25,26}$ Recently, it has been widely used as a matrix to fabricate nanostructured hybrid anodes to boost the performance of $\mathrm{CuO}$ anode electrodes..$^{13,22-24}$ In our previous work, we reported an ultra-short rice-like CuO nanorods/RGO composite with a high reversible specific capacity of 762.6 $\mathrm{mA} \mathrm{h} \mathrm{g}^{-1}$ at a charge rate of $100 \mathrm{~mA} \mathrm{~g}^{-1}$ after 100 cycles. $^{27} \mathrm{We}$ found that the small dimensional $\mathrm{CuO}$ nanorods combined with conductive graphene sheets exhibited high specific capacity and good cyclic stability. However, it is still a big challenge to obtain the high-rate capability of CuO-based anodes.

Recently, many studies demonstrated that porous structures provide significant advantages to enhance the lithium storage performance of electroactive materials because of its large surface area and high porosity, which can restrain the huge volume change and offer good surface contact between the electrolyte and the electrode. ${ }^{11,28-30}$ For example, Wang et al. ${ }^{6}$ synthesized porous $\mathrm{CuO}$ nanorods and achieved $654 \mathrm{~mA} \mathrm{~h}^{-1}$ at a charge rate of $0.5 \mathrm{C}$ for 200 cycles. Yuan et al. ${ }^{31}$ fabricated $3 \mathrm{D}$ porous $\mathrm{SnO}_{2}-\mathrm{Fe}_{2} \mathrm{O}_{3}$ composite via an electrostatic spray deposition technique; they show enhanced lithium storage performance because of the synergistic effect between the $\mathrm{Fe}_{2} \mathrm{O}_{3}$ and the $\mathrm{SnO}_{2}$, and also the $3 \mathrm{D}$ hierarchical porous structure. Currently, Mohapatra et al. ${ }^{32}$ prepared mulberry-like porous $\mathrm{CuO}$ and $\mathrm{CuO}$ nanoplate, which found that the mulberry-like 
porous $\mathrm{CuO}$ electrode exhibited higher capacity in starting several cycles than that of the $\mathrm{CuO}$ nanoplate electrode because of the porous architecture of the electrode. However, this $\mathrm{CuO}$ sample showed poor cyclic stability due to its structural instability during cycling. Thus, it is expected that porous $\mathrm{CuO}$ nanostructures modified via the flexible and conductive graphene sheets could display favorable anodic performances for LIB anode. However, to this day, the reports about the preparation of porous CuO-based anodes for LIBs are still rare, especially for self-assembly porous $\mathrm{CuO} /$ graphene composite.

In this work, a facile two-step thermal treatment procedure for the synthesis of porous CuO-NSs decorated on RGO nanosheets was firstly reported. The porous CuO-NSs with a homogeneous size of $\sim 100 \mathrm{~nm}$, are assembled by the ultra-fine nanoparticles of $\mathrm{CuO}$ with a size of around $10-15 \mathrm{~nm}$. The asprepared CuO-NSs/RGO composite possesses the combined merits of graphene and the porous shape of $\mathrm{CuO}$, which can display the following advantages: (i) the electrical conductivity and structural stability of the porous CuO-NSs/RGO can be enhanced through the conductive and flexible graphene; (ii) the porous characteristics of $\mathrm{CuO}$ can shorten the diffusion distance for $\mathrm{Li}^{+}$ions and buffer the large volume change during cycling. Hence, the porous CuO-NSs/RGO composite can deliver large reversible specific capacity, long cycling lifespan, and outstanding high-rate performance for lithium storage.

\section{Results and discussion}

The synthesis process of the porous CuO-NSs/RGO composite is shown in Fig. 1. The preparation of the solution of $\mathrm{Cu}^{2+}$ ions in toluene in detail is illustrated in Fig. S1 of ESI Materials. $\dagger$ Firstly, the solution of $\mathrm{Cu}^{2+}$ ions in toluene was prepared by a modification of the procedure reported by Yang et al. ${ }^{34}$ In this case, cetyltrimethyl ammonium bromide (CTAB) was served as stabilizer to promote the dispersion of GO in toluene to form a homogeneous GO suspension. Taking advantage of the abundant oxygen functional groups, $\mathrm{Cu}^{2+}$ ions can be tightly adsorbed on the surface of GO sheets due to electrostatic interaction. Subsequently, $\mathrm{Cu}_{2} \mathrm{O}$ nanoparticles were grown uniformly on RGO sheets via chemical reduction with hydrazine hydrate as reductant. Finally, the porous CuO-NSs/RGO composite was obtained after thermally treated the resultant $\mathrm{Cu}_{2} \mathrm{O} / \mathrm{RGO}$ composite under air atmosphere.

The crystalline structure of the as-made products was investigated by XRD characterization, which is given in Fig. 2a. After chemical reduction, the pattern shows that cubic $\mathrm{Cu}_{2} \mathrm{O}$

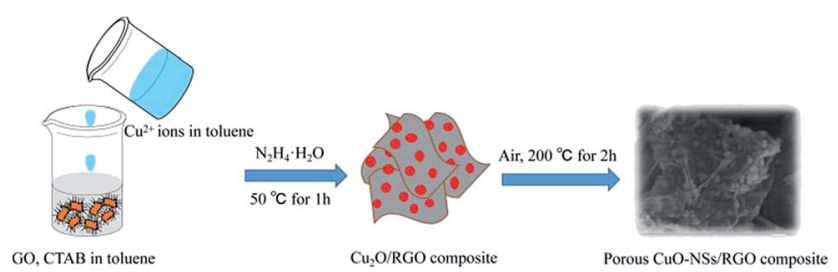

Fig. 1 Schematic diagram for illustrating the synthesis procedure of porous $\mathrm{CuO}-\mathrm{NSs} / \mathrm{RGO}$ composite.
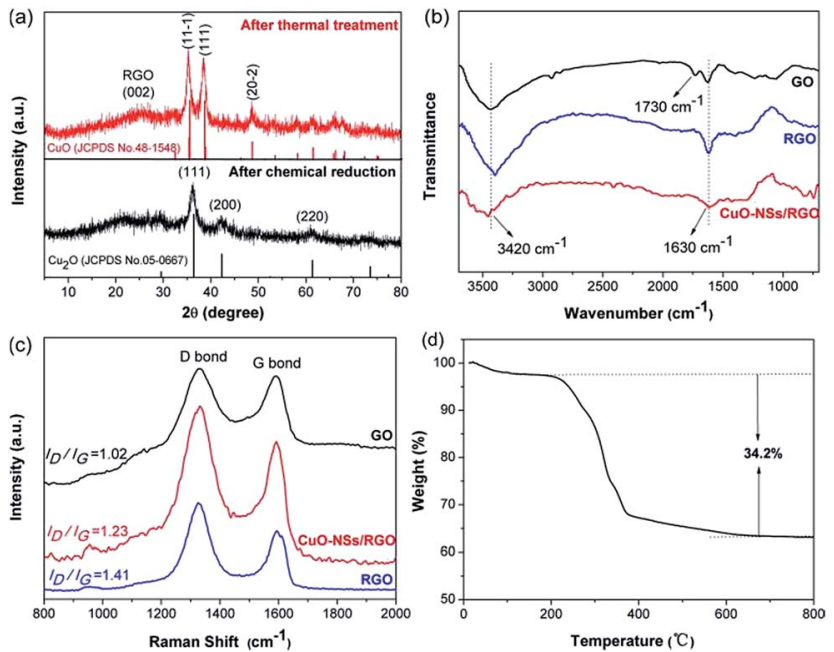

Fig. 2 (a) XRD patterns of the as-prepared samples; (b) FTIR and (c) Raman spectra of GO, RGO, and CuO-NSs/RGO composite; (d) TGA curves of the CuO-NSs/RGO composite.

phase (JCPDS No. 05-0667) was formed. After thermal treatment, the pattern verifies the formation of monoclinic $\mathrm{CuO}$ phase (JCPDS No. 48-1548). The diffraction peak at $\sim 26^{\circ}$ can be assigned to RGO nanosheets, and no characteristic diffraction peak of GO at $\sim 10^{\circ}$ can be observed (Fig. S2 of ESI Materials $\dagger$ ), indicating the effective reduction from GO to RGO by hydrazine hydrate. The structure change of the samples can be further investigated by FTIR and Raman spectroscopy, which is shown in Fig. $2 \mathrm{~b}$ and c. As presented in Fig. $2 \mathrm{~b}$, the adsorption peaks at 3420,1730 , and $1630 \mathrm{~cm}^{-1}$ of the GO sample could be assigned to the characteristic peak of hydroxy stretching vibrations, carbonyl stretching vibrations, and unoxidized graphitic domains or adsorbed water, respectively, consisting well with previous reports. ${ }^{35,36}$ As regards the CuO-NSs/RGO composite, the adsorption peak of hydroxy was attenuated, and the peak of carbonyl groups was disappeared in both RGO and CuO-NSs/ RGO composite, indicating that GO could be effectively reduced into RGO. The significant structural change of GO after the two-step thermal treatment procedure was further demonstrated by Raman spectroscopy. Fig. 2c shows that two main typical peaks located at $\sim 1580$ and $\sim 1350 \mathrm{~cm}^{-1}$ in the Raman spectra of the three samples are assigned to the and $\mathrm{G}$ band and $\mathrm{D}$, respectively. It is generally believed that a high increased value of $I_{\mathrm{D}} / I_{\mathrm{G}}$ (intensity ratio of $\mathrm{D}$ band to $\mathrm{G}$ band) results in more disordered carbon atoms in graphene materials. ${ }^{35-38}$ The Raman spectra of the as-synthesized products show the higher $I_{\mathrm{D}} / I_{\mathrm{G}}$ values of CuO-NSs/RGO composite (1.23) and RGO nanosheets (1.41) than that of in GO (1.02), suggesting that the structure of GO has been changed significantly after the twostep thermal treatment, and a high defective nature of $\mathrm{CuO}$ NSs/RGO composite was obtained. The existence of defects can be helpful to improve the ability of interfacial lithium storage capacity of LIBs.

The amount of graphene in the CuO-NSs/RGO composite was calculated by TGA, as presented in Fig. 2 d. The small weight lose about $2.5 \%$ below $200{ }^{\circ} \mathrm{C}$ could be ascribed to the 
evaporation of physical absorbed water. The weight decreased dramatically in the range from 250 to $400{ }^{\circ} \mathrm{C}$ and then remained unchanged till $800{ }^{\circ} \mathrm{C}$, which could be attributed to the oxidation of the RGO nanosheets upon exposure to air. Thus the content of RGO in the CuO-NSs/RGO composite is evaluated as $\sim 34.2 \mathrm{wt} \%$. Such an acceptable amount of RGO nanosheets in the CuO-NSs/RGO composite, on the one hand, endows the composite a good electronic conductivity, and effectively suppresses the pulverization and aggregation of CuO-NSs during the cycling process. On the other hand, the sufficient $\mathrm{CuO}$ in the composite ensures high capacity.

The morphologic characteristics of the as-prepared $\mathrm{CuO}-$ NSs/RGO composite are shown in Fig. 3. SEM images (Fig. 3ac) reveal that the porous CuO-NSs and RGO nanosheets in this composite are interconnected well, and the porous CuO-NSs are uniformly supported on or wrapped in the RGO nanosheets. The SEM images of the $\mathrm{Cu}_{2} \mathrm{O} / \mathrm{RGO}$ composite (Fig. S3 of ESI Materials $\dagger$ ) show a similar morphology as the CuO-NSs/RGO composite, indicating no significant changes for the morphologies of the products after chemical reduction and thermal treatment. Fig. 4 shows the elemental mapping of the CuO-NSs/ RGO composite. It can be obviously observed that the elements $\mathrm{C}$ (Fig. 4b), O (Fig. 4c) and $\mathrm{Cu}$ (Fig. 4d) are uniformly distributed on the composite. EDS pattern of the composite, as shown in Fig. $\mathbf{S} 4, \dagger$ also confirms the existence of the three elements.

The detailed micromorphologies of the products were investigated by TEM and are presented in Fig. 5. Fig. 5a and b show that the pristine $\mathrm{CuO}$ is randomly aggregated by ultrafine $\mathrm{CuO}$ nanoparticles due to the high surface energy, ${ }^{39}$ and only few spherical secondary aggregates can be found. Fig. 5c shows that the size of the primary CuO nanoparticle is $\sim 30 \mathrm{~nm}$.

The porous feature of the CuO-NSs/RGO composite was observed in Fig. 5d and e, which displays that the porous $\mathrm{CuO}-$ NSs have a homogeneous size of $\sim 100 \mathrm{~nm}$, and are homogeneously decorated on RGO nanosheets. Simultaneously, Fig. 5e reveals that the porous CuO-NSs were loosely and orderly aggregated by the primary ultra-fine $\mathrm{CuO}$ nanoparticles with high surface energies to form porous structured secondary aggregates. For one thing, such unique porous nanostructured morphology can buffer the volume expansion and contraction during lithiation and delithiation process. More importantly, the structural stability of porous CuO-NSs could be significantly enhanced by introducing the flexible RGO nanosheets. For another, the multi-dimensional channels for electron transport and $\mathrm{Li}^{+}$ion diffusion can be offered, so the structure of $\mathrm{CuO}$ NSs/RGO composite can be beneficial to facilitate the permeation of electrolyte and apparently improve the rate capability.
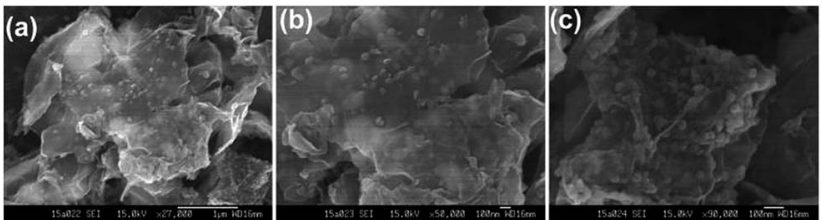

Fig. 3 (a-c) Typical SEM images of the as-prepared CuO-NSs/RGO composite.

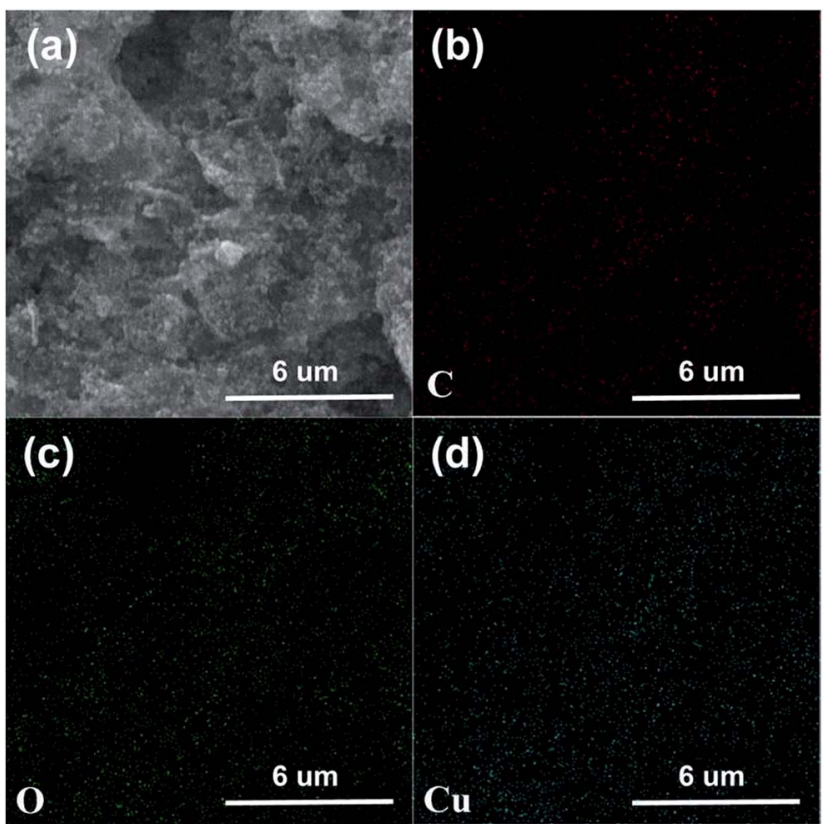

Fig. 4 Elemental mapping of the as-synthesized porous CuO-NSs/ RGO composite.

The HRTEM image of individual ultra-fine $\mathrm{CuO}$ nanoparticle in the composite is presented in Fig. 5f, which shows that the primary nanosized $\mathrm{CuO}$ particles have a diameter of around $15 \mathrm{~nm}$. The distinct lattice distances of 0.252 and $0.232 \mathrm{~nm}$, corresponding to the (11-1) and (111) lattice planes of monoclinic $\mathrm{CuO}$, respectively, which is coincide well with the XRD peaks (Fig. 2a). Noting that the size of the individual $\mathrm{CuO}$ nanoparticle in CuO-NSs/RGO composite is smaller than that of in pristine CuO. The possible explanation is that, after the mix of the solution of $\mathrm{Cu}^{2+}$ ions in toluene and the GO suspension, $\mathrm{Cu}^{2+}$ ions were adsorbed on the surface of GO sheets due to the electrostatic interactions, thus the concentration of the free $\mathrm{Cu}^{2+}$ ions in toluene was decreased. The adsorbed $\mathrm{Cu}^{2+}$ ions on

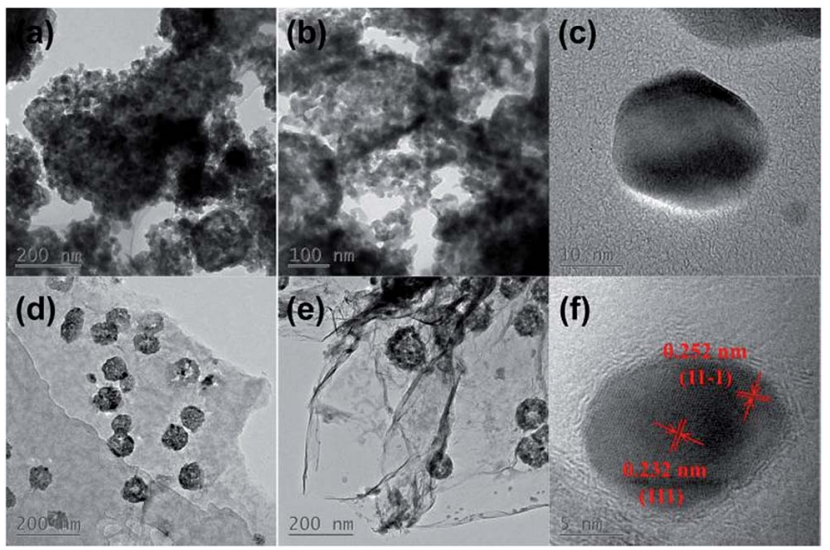

Fig. 5 ( $a$ and b) TEM images of the pristine CuO; (c) HRTEM image of an individual $\mathrm{CuO}$ nanoparticle in pristine $\mathrm{CuO}$; ( $d$ and e) TEM images of the as-prepared CuO-NSs/RGO composite; (f) HRTEM image of an individual $\mathrm{CuO}$ nanoparticle on RGO. 
GO sheet could act as the heterogeneous nucleation sites so that the size of the individual $\mathrm{CuO}$ nanoparticle in the CuO-NSs/RGO composite is smaller than that of in pristine $\mathrm{CuO}$.

$\mathrm{N}_{2}$ sorption measurements were operated to further analyze the pore structure of the as-synthesized samples. Fig. 6a shows the $\mathrm{N}_{2}$ adsorption-desorption isotherms of the samples, which are of type IV with a distinct hysteresis, indicating the porous features of the products. The specific surface area of the CuONSs/RGO composite calculated by Brunauer-Emmett-Teller (BET) method is $61.9 \mathrm{~m}^{2} \mathrm{~g}^{-1}$, and the Barrett-Joyner-Halenda (BJH) desorption cumulative pore volume of the CuO-NSs/RGO composite is $0.28 \mathrm{~cm}^{3} \mathrm{~g}^{-1}$, which is larger than those of in pristine $\mathrm{CuO}\left(15.6 \mathrm{~m}^{2} \mathrm{~g}^{-1}, 0.09 \mathrm{~cm}^{3} \mathrm{~g}^{-1}\right)$ and RGO nanosheets $\left(4.7 \mathrm{~m}^{2} \mathrm{~g}^{-1}, 0.05 \mathrm{~cm}^{3} \mathrm{~g}^{-1}\right)$. The CuO-NSs/RGO composite possesses large specific surface area, which could be ascribed to the synergistic effect between the porous CuO-NSs and the RGO nanosheets. The RGO nanosheets served as substrate for the ordered agglomeration of primary $\mathrm{CuO}$ nanoparticles to form porous spherical secondary agglomerates. At the same time, the re-stacking of RGO nanosheets could be inhibited by the porous CuO-NSs, resulting in a high specific surface area of the porous CuO-NSs/RGO composite. However, pristine $\mathrm{CuO}$ was severely and disorderly agglomerated due to its high surface energy and small size effect as demonstrated in Fig. $5 \mathrm{a}$ and b, and the small specific surface area of RGO is mainly caused by the re-stacking of graphene nanosheets owing to the recovery of $\pi-\pi$ conjugated system from the GO sheets upon chemical reduction. ${ }^{\mathbf{4 0}}$

The pore size distribution curves of the as-prepared products were obtained using BJH model, which are shown in Fig. 6b. The porous CuO-NSs/RGO composite exhibits a small portion of pores centred at $2.6 \mathrm{~nm}$ and a majority of pores in the range of $10-60 \mathrm{~nm}$. The pores size centred at $2.6 \mathrm{~nm}$ may be generated by the slit of CuO-NSs originated from the self-assembly of ultrafine nanoparticles of $\mathrm{CuO}$, while the wide pores size distribution of 10-60 $\mathrm{nm}$ could be attributed to the pore formation between the porous CuO-NSs and the RGO sheets. ${ }^{\mathbf{4 1}}$ By contrast, the pore size distribution curves of pristine $\mathrm{CuO}$ and RGO demonstrate the small amount of meso- and macropores formed. So it is believed that the lithium storage performances of the porous CuO-NSs/RGO composite could be significantly enhanced because of their porous characteristics.

The electrochemical performance of the porous CuO-NSs/ RGO composite anode was firstly detected by cyclic voltammetry (CV) tests, which is displayed in Fig. 7a. In the first cathodic sweep, three well-resolved reduction peaks at $\sim 2.0,1.2$, and
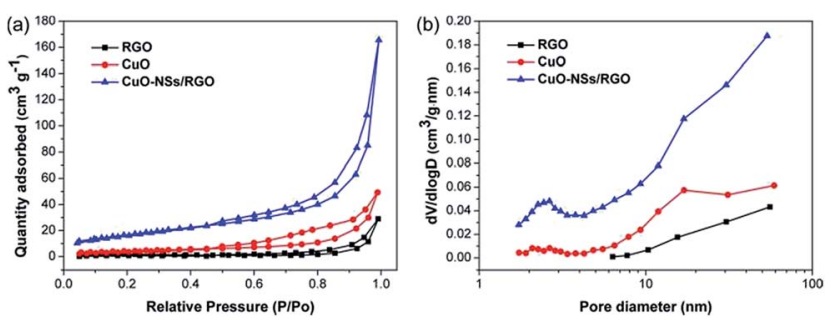

Fig. 6 (a) $\mathrm{N}_{2}$ adsorption-desorption isotherms and (b) BJH pore size distribution curves of the as-synthesized samples.
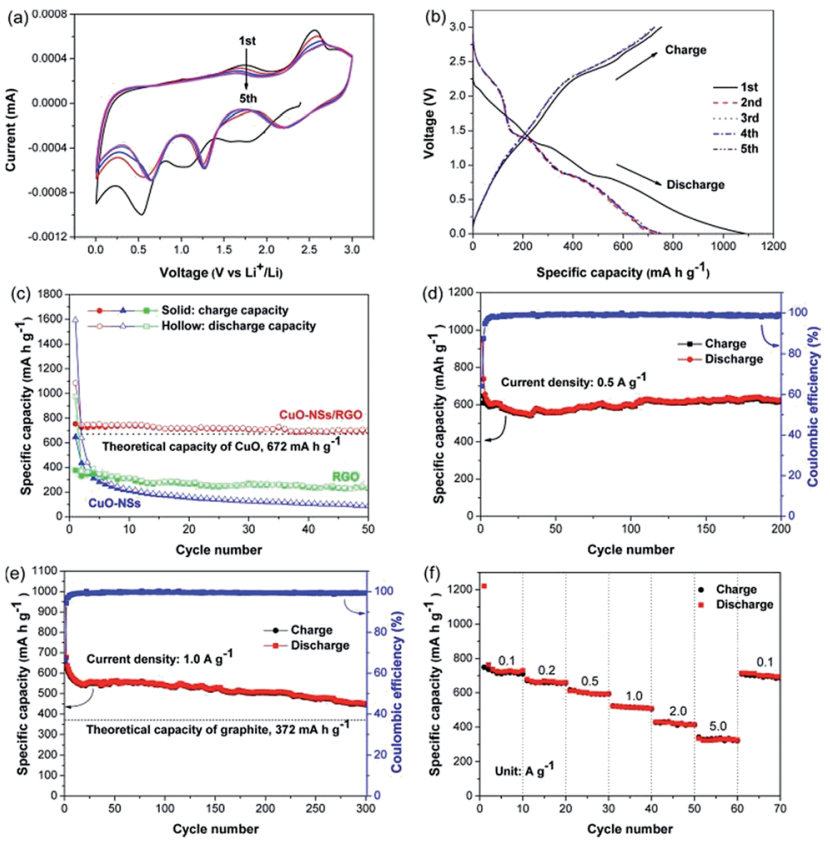

Fig. 7 (a) CV curves of the CuO-NSs/RGO composite at a scanning rate of $0.5 \mathrm{mV} \mathrm{s}^{-1}$; (b) discharge-charge profiles of the CuO-NSs/RGO composite at a charge rate of $0.1 \mathrm{~A} \mathrm{~g}^{-1}$; (c) cycling performance of $\mathrm{RGO}$, pristine $\mathrm{CuO}$, and $\mathrm{CuO}-\mathrm{NSs} / \mathrm{RGO}$ composite at a charge rate of $0.1 \mathrm{~A} \mathrm{~g}^{-1}$; ( $\mathrm{d}$ and e) cycling performance and coulombic efficiency of the CuO-NSs/RGO composite at different charge rates; (f) rate capability of the $\mathrm{CuO}-\mathrm{NSs} / \mathrm{RGO}$ composite at different charge rates range from 0.1 to $5 \mathrm{~A} \mathrm{~g}^{-1}$.

$0.5 \mathrm{~V}$ were attributed to the formation of $\mathrm{Cu}_{1-x}^{\mathrm{II}} \mathrm{Cu}_{x}^{\mathrm{I}} \mathrm{O}_{1-x / 2}$ phase, the creation of $\mathrm{Cu}_{2} \mathrm{O}$ phase, and then decomposed into $\mathrm{Cu}$ and $\mathrm{Li}_{2} \mathrm{O}$, respectively. ${ }^{10,13,16}$ The reduction peak at $\sim 0.5 \mathrm{~V}$ also involves the growth of a solid electrolyte interface (SEI) film over the surface of electrode. In the following anodic sweep, three distinctive oxidation peaks at around 1.6, 2.5 (strong) and $2.9 \mathrm{~V}$ (weak) can be observed, which could be assigned to the partial decomposition of SEI layer, the formation of $\mathrm{Cu}_{2} \mathrm{O}$, and the conversion of $\mathrm{Cu}_{2} \mathrm{O}$ into $\mathrm{CuO}$, respectively. ${ }^{11}$ In the following scanning, the three reduction peaks overlap very well starting from the second cycle, however, with little shift to high potential region because of the formation of SEI film resulted by the partial decomposition of electrolyte, ${ }^{\mathbf{4 2 , 4 3}}$ indicating that the overpotential of discharge process gradually decreased within the increased cycle number. For the three oxidation peaks, the individual peak area is decreased, implying reversible capacity loss. Fig. 7b depicts the initial five cycles galvanostatic charging and discharging curves of the porous CuO-NSs/RGO composite at $0.1 \mathrm{~A} \mathrm{~g}^{-1}$. Plateaus at around $2.0,1.2$, and $0.7 \mathrm{~V}$ in the initial discharging curve, and at around 1.6, 2.5, and $2.9 \mathrm{~V}$ during the first charging curve indicate a multi-step electrochemical reactions of $\mathrm{CuO}^{\mathbf{4 4}}$ The first-cycle discharge capacity of the CuONSs/RGO composite is $1085.3 \mathrm{~mA} \mathrm{~h} \mathrm{~g}{ }^{-1}$ with a reversible capacity of $753.3 \mathrm{~mA} \mathrm{~h} \mathrm{~g}{ }^{-1}$, corresponding to a first-cycle coulombic efficiency of $69.4 \%$. The high initial irreversible capacity loss of the porous CuO-NSs/RGO composite is mainly attributed to the formation of SEI layer, the irreversible 
insertion of $\mathrm{Li}^{+}$ions into RGO scaffolds, and the partial decomposition of electrolyte. ${ }^{\mathbf{1 0 , 1 6}}$ It should be noting that the conversion of $\mathrm{Cu}$ into $\mathrm{CuO}$ is incomplete, which causes a number of $\mathrm{Li}^{+}$ions could not participate in the subsequent electrochemical reaction, leading to the irreversible capacity loss. ${ }^{45,46}$ Fig. $7 \mathrm{c}$ depicts the comparative cyclic performance of the porous CuO-NSs/RGO composite, pristine CuO, and RGO sheets electrode at $0.1 \mathrm{~A} \mathrm{~g}^{-1}$. The pristine $\mathrm{CuO}$ electrode can deliver a high first-cycle discharge capacity of $1593.5 \mathrm{~mA} \mathrm{~h} \mathrm{~g}^{-1}$ with a reversible capacity of $646.2 \mathrm{~mA} \mathrm{~h} \mathrm{~g}^{-1}$. But the discharge capacity sharply decreased to $88 \mathrm{~mA} \mathrm{~h} \mathrm{~g}^{-1}$ after 50 cycles. This result could be attributed to the structure collapse and/or pulverization of $\mathrm{CuO}$ during charge/discharge process, which might make the electroactive $\mathrm{CuO}$ material fall away from the current collector. As evidenced in Fig. S5 of ESI Materials, $\dagger$ the separators were peeled down from the cells after 50 cycles. It can be observed that the separator obtained from a pristine $\mathrm{CuO}$ cell was coated with brown materials. However, the separator from a CuO-NSs/RGO composite cell still remains the same color as the pristine separator, indicating the structural stability and integrity of the porous CuO-NSs/RGO electrode. For the RGO electrode, a high first-cycle discharge capacity of $976.4 \mathrm{~mA} \mathrm{~h} \mathrm{~g}^{-1}$ was delivered. However, the charge capacity was gradually decreased from 1st cycle $\left(376.2 \mathrm{~mA} \mathrm{~h} \mathrm{~g}^{-1}\right)$ to 50 th cycle $(228.5$

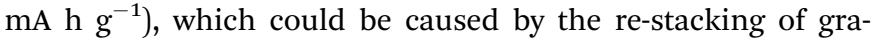
phene nanosheets. By contrast, the porous CuO-NSs/RGO electrode exhibits a higher reversible capacity, and a lower irreversible capacity loss than those of the pristine $\mathrm{CuO}$ electrode. The coulombic efficiency of the CuO-NSs/RGO electrode at first cycle is $69.4 \%$, and then increased dramatically to $96.9 \%$ in the 2nd cycle and near $\sim 99 \%$ after the subsequent cycles, indicating its remarkable reversibility. The capacity retention of $90.6 \%$ was achieved after 50 cycles $\left(692.8 \mathrm{~mA} \mathrm{~h} \mathrm{~g}^{-1}\right)$, suggesting the stable cyclability of the porous CuO-NSs/RGO composite electrode.

To further demonstrate the stable cycling performance of the porous CuO-NSs/RGO composite electrode, the cells were measured at a high charge rate of 0.5 and $1.0 \mathrm{~A} \mathrm{~g}^{-1}$ with prolonged cycles. Fig. $7 \mathrm{~d}$ and e show that the initial discharge and charge capacities at $0.5 \mathrm{~A} \mathrm{~g}^{-1}$ are 948.8 and $608.1 \mathrm{~mA} \mathrm{~h} \mathrm{~g}^{-1}$, and at $1 \mathrm{~A} \mathrm{~g}^{-1}$ are 968.6 and $643.7 \mathrm{~mA} \mathrm{~h} \mathrm{~g}^{-1}$, with a first-cycle coulombic efficiency of $64.1 \%$ and $66.5 \%$, respectively. The CuO-NSs/RGO composite electrode exhibited perfect cycling stability, with no capacity lose at $0.5 \mathrm{~A} \mathrm{~g}^{-1}$ after 200 cycles $(616.2$ $\mathrm{mA} \mathrm{h} \mathrm{g}{ }^{-1}$ for the 200th cycle). Even at high charge rate of $1 \mathrm{Ag}^{-1}$, the reversible capacity after 300 cycles can still achieve $446 \mathrm{~mA} \mathrm{~h}$ $\mathrm{g}^{-1}$, demonstrating its good specific capacity retention and enhanced cyclic stability. The morphology of the composite after 200 cycles at $0.5 \mathrm{~A} \mathrm{~g}^{-1}$ was observed through ex situ SEM. Fig. S6 $†$ presents the SEM images of the composite before and after cycling. Noting that the composite can maintain its structural integrity after 200 cycles, suggesting the structural stability of the composite.

Fig. $7 \mathrm{f}$ shows the rate capability of the porous CuO-NSs/RGO composite electrode. The battery was cycled at different charge rates range from 0.1 to $5.0 \mathrm{~A} \mathrm{~g}^{-1}$, and maintained at 10 cycles for each charge rate. It was found that the porous CuO-NSs/RGO composite electrode exhibited an outstanding high-rate capability. The average reversible capacities at $0.1,0.2,0.5,1.0$, and $2.0 \mathrm{~A} \mathrm{~g}^{-1}$ are $720.8,659.4,599.1,514.5$, and $419.9 \mathrm{~mA} \mathrm{~h} \mathrm{~g}^{-1}$ respectively. Even at a high charge rate of $5 \mathrm{~A} \mathrm{~g}^{-1}$, an average reversible capacity of $329.4 \mathrm{~mA} \mathrm{~h} \mathrm{~g}^{-1}$ can still be obtained. When the current density was reset to $0.1 \mathrm{~A} \mathrm{~g}^{-1}$, an average reversible capacity of $696.1 \mathrm{~mA} \mathrm{~h} \mathrm{~g}^{-1}$ was still obtained, suggesting good reversibility. Compared with some other CuObased anodes as presented in Table S1, $\uparrow$ the porous CuO-NSs/ RGO composite possesses excellent rate performance when served as a LIB anode. The prominent lithium storage properties of the composite could be mainly attributed to the synergistic effect between the porous CuO-NSs and RGO nanosheets, as schematically illustrated in Fig. 8. First, the porous architecture of CuO-NSs was given by the self-assembly of ultra-fine $\mathrm{CuO}$ nanoparticles, which is beneficial to the electrolyte permeation from outside into inside of CuO. Furthermore, the $\mathrm{Li}^{+}$ion diffusion length and the electron transfer distance could also be shortened due to the porous structure, resulting in an ability of rapid charge/discharge. Additionally, a good conductive network for the fast electron transfer could be formed by the introduction of RGO nanosheets, which also serve as cushions to relieve the stress caused by the huge volume change of $\mathrm{CuO}$ NSs upon cycling, thus enhancing the structural integrity and stability of the composite.

To further understand the reason why the composite electrode exhibited such good lithium storage performances, in particular, the high-rate capability, a comparative study of EIS between the characteristics of pristine $\mathrm{CuO}$ electrode and the porous CuO-NSs/RGO composite electrode has been proposed. Nyquist plots of the pristine $\mathrm{CuO}$ and the porous CuO-NSs/RGO composite electrode obtained at full charge state after five cycles and are shown in Fig. 9a. It can be seen from the electrochemical impedance spectra (EIS) that the shape of the Nyquist plots is similar, a depressed semicircle followed by a sloped line. The depressed semicircle in the high- and mediumfrequency region represents the charge-transfer process, involving the total resistance come from the SEI film and the interior of the electrode. While the sloped line in low frequencies associates with $\mathrm{Li}^{+}$ion diffusion within the electrode. ${ }^{10,15} \mathrm{As}$ seen in Fig. 9a, the depressed semicircle of the CuO-NSs/RGO electrode is far smaller than that of pristine $\mathrm{CuO}$ electrode, suggesting a low resistance for the CuO-NSs/RGO electrode. The relationship between $Z^{\prime}$ and $\omega^{-1 / 2}$ after 5 cycles is presented in Fig. $9 \mathrm{~b} . Z^{\prime}$ is the real part of the impedance spectra, and $\omega$ is the angular frequency, provided by $\omega=2 \pi f$. The low slope of $Z^{\prime}$ versus $\omega^{-1 / 2}$ indicates a high $\mathrm{Li}^{+}$ion diffusion rate. ${ }^{12,47}$ It can

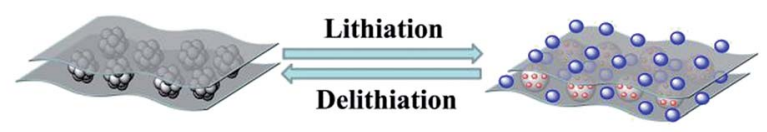

RGO nanosheet Porous CuO-NSs $\mathrm{Li}_{2} \mathrm{O} \odot \mathrm{Li}_{\mathrm{x}} \mathrm{C} \quad \odot \mathrm{Cu}$ nanoparticle

Fig. 8 Schematic illustration of the lithiation/delithiation process of the porous $\mathrm{CuO}-\mathrm{NSs} / \mathrm{RGO}$ composite. 

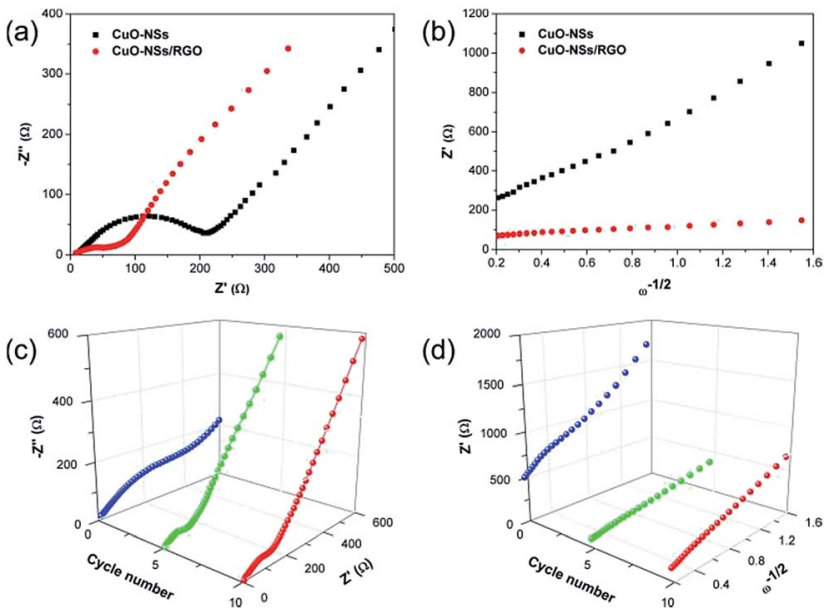

(d)

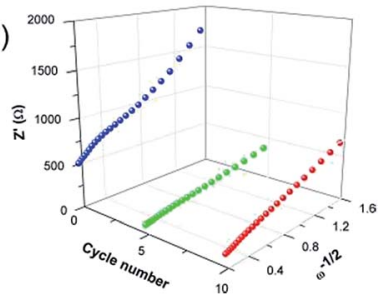

Fig. 9 (a) Nyquist plots of pristine $\mathrm{CuO}$ and the $\mathrm{CuO}-\mathrm{NSs} / \mathrm{RGO}$ composite after 5 cycles; (b) the relationship between $Z^{\prime}$ and $\omega^{-1 / 2}$; (c) 3D Nyquist plots of the CuO-NSs/RGO composite and (d) the relationship between $Z^{\prime}$ and $\omega^{-1 / 2}$ before and after 5 and 10 cycles.

be seen that the slope of the porous CuO-NSs/RGO electrode is much lower than the pristine CuO electrode, suggesting a high $\mathrm{Li}^{+}$ion diffusion rate was achieved in the CuO-NSs/RGO electrode, which could be attributed to the well-conductive network in the composite with the introduction of high-conductivity of RGO nanosheets. Thus, the total resistance of the CuO-NSs/ RGO electrode could be reduced and the migration rate of $\mathrm{Li}^{+}$ions could also be significantly enhanced. Fig. 9c shows a comparative EIS analysis of the porous CuO-NSs/RGO composite before and after different cycles. It can be observed that the depressed semicircle of the CuO-NSs/RGO electrode is dramatically reduced after 5 cycles due to the electrochemical activation process of the electrode. However, it becomes larger after 10 cycles, which could be caused by the continuous growth of the SEI film. ${ }^{50,51}$ Fig. 9d presents the corresponding relationship between $Z^{\prime}$ and $\omega^{-1 / 2}$. The slope of $Z^{\prime}$ versus $\omega^{-1 / 2}$ after 5 and 10 cycles is much lower than that of before cycling, indicating that the fast $\mathrm{Li}^{+}$ion diffusion rate is achieved after cycling. ${ }^{52}$

To verify the viability of the porous CuO-NSs/RGO composite as prospective anode for LIBs, CuO-NSs/RGO//LFP full cells were assembled by using the porous CuO-NSs/RGO composite anodes and commercial LFP cathodes. Fig. 10 depicts the electrochemical performance of the LFP half-cell and the CuONSs/RGO//LFP full cell. The cycling performance of the LFP halfcell was firstly examined, as shown in Fig. 10a. The reversible specific capacity gradually decreased from 1 st cycle $(132.4 \mathrm{~mA} \mathrm{~h}$ $\left.\mathrm{g}^{-1}\right)$ to 30 th cycle $\left(83.6 \mathrm{~mA} \mathrm{~h} \mathrm{~g}^{-1}\right)$ at $0.5 \mathrm{C}\left(1 \mathrm{C}=170 \mathrm{~mA} \mathrm{~g}^{-1}\right)$. Fig. 10b shows the rate performance of the LFP half-cell. The LFP half-cell delivers a reversible capacity of $160.8 \mathrm{~mA} \mathrm{~h} \mathrm{~g}^{-1}$ at $0.5 \mathrm{C}$, when the charge rate increased to $2 \mathrm{C}$, the capacity drastically decreased to $\sim 90 \mathrm{~mA} \mathrm{~h} \mathrm{~g}{ }^{-1}$. Fig. 10c presents the initial galvanostatic charge and discharge curves of the CuO-NSs/ $\mathrm{RGO} / / \mathrm{LFP}$ full cell at $0.5 \mathrm{C}$ cycling between 0.8 and $3.8 \mathrm{~V}$. The full cell gives a first charging and discharging capacities of

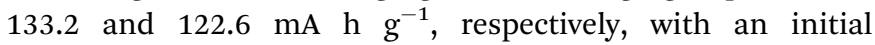
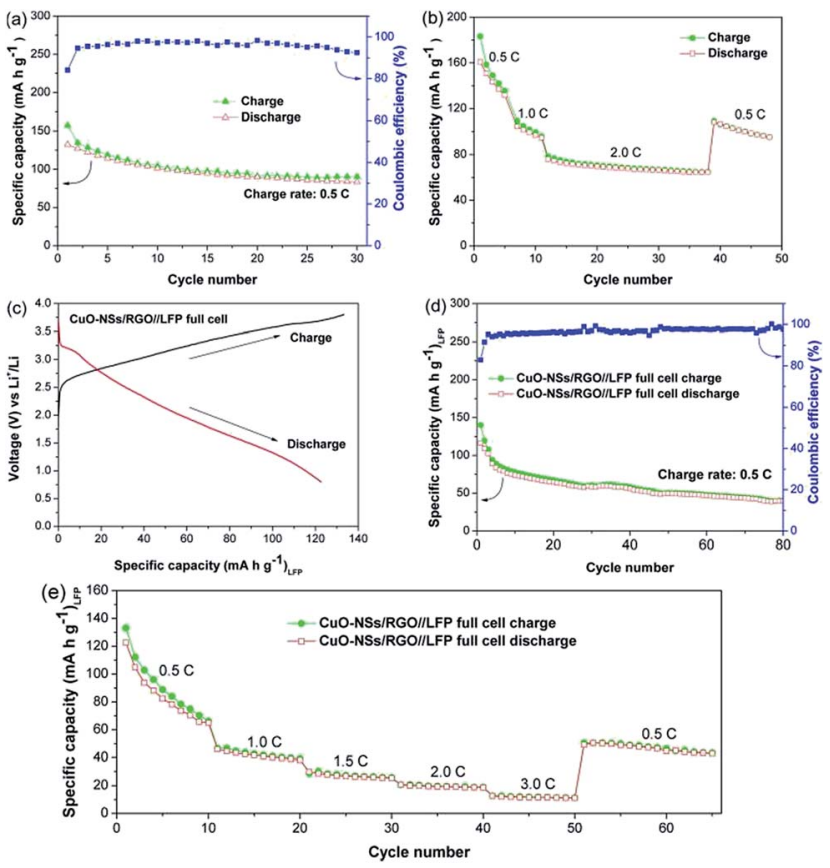

Fig. 10 (a) Cycling performance of the LFP half cell at $0.5 \mathrm{C}$; (b) rate performance of the LFP half cell at different charge rates range from 0.5 to $2 \mathrm{C}$; (c) discharge-charge profiles of the CuO-NSs/RGO//LFP full cell at $0.5 \mathrm{C}$; (d) cycling performance and coulombic efficiency of the $\mathrm{CuO}-\mathrm{NS} / \mathrm{RGO} / / \mathrm{LFP}$ full cell at $0.5 \mathrm{C}$; (e) rate capability of the CuO$\mathrm{NSs} / \mathrm{RGO} / / \mathrm{LFP}$ full cell at different charge rates range from 0.5 to $3 \mathrm{C}$.

coulombic efficiency of as high as $92 \%$. It should be pointed out that the electrochemical activation process of anode electrode remarkably enhanced the coulombic efficiency of the CuO-NSs/ $\mathrm{RGO//LFP} \mathrm{full} \mathrm{cell} \mathrm{because} \mathrm{it} \mathrm{can} \mathrm{compensate} \mathrm{the} \mathrm{loss} \mathrm{of} \mathrm{lithium}$ during the formation of SEI layer or other side reactions. The cycling performance of the CuO-NSs/RGO//LFP full cell at $0.5 \mathrm{C}$ is shown in Fig. 10d. The full cell exhibited large capacity loss from 116.3 to $39.6 \mathrm{~mA} \mathrm{~h} \mathrm{~g}^{-1}$ after 80 cycles, which could be mainly caused by the restricted source of $\mathrm{Li}$ from cathode side (cathode limited). ${ }^{48}$

The rate capability of the CuO-NSs/RGO//LFP full cell was also examined (Fig. 10e). The full cell delivered an average reversible capacities of 84.5, 41.6, 27.0, 19.4 and $11.6 \mathrm{~mA} \mathrm{~h} \mathrm{~g}^{-1}$ at $0.5,1.0,1.5,2.0$ and $3.0 \mathrm{C}$, respectively. It is found that the rate property of the CuO-NSs/RGO//LFP full cell is not in agreement with those of CuO-NSs/RGO half-cell but consistent well with those of LFP half-cell. This result could be attributed to the following reasons. On the one hand, lithium source is limited by the LFP cathode in the full cell. But for the half-cell, Li foil as the counter/reference electrode can provide excess $\mathrm{Li}^{+}$ions, which is in favor of improving the electrochemical reaction kinetics of the cell. On the other hand, it is believed that the rate property of the CuO-NSs/RGO//LFP full cell is affected by both of CuO-NSs/RGO anode based on the conversion reaction and LFP cathode based on the insertion/deinsertion mechanism..$^{49}$ Thus, the incompatibilities of the conversion reaction ratio and the insertion/deinsertion degree make the CuO-NSs/RGO//LFP full cell an unsatisfied rate property. 


\section{Conclusions}

In summary, porous CuO-NSs/RGO composite was synthesized through a two-step thermal treatment procedure. It was found that the porous $\mathrm{CuO}-\mathrm{NSs}$ are assembled by the ultra-fine $\mathrm{CuO}$ nanoparticles, and are homogeneously decorated on RGO nanosheets. The as-made porous CuO-NSs/RGO composite was used as a LIB anode, which exhibited superior anodic properties, including high reversible specific capacity, enhanced cyclic stability, and outstanding high-rate capability, which could be ascribed to the synergistic effect between the porous CuO-NSs and the flexible and well-conductive RGO nanosheets. The feasibility of the porous CuO-NSs/RGO composite as anode for LIBs was investigated with commercial LFP cathode. These results show that by further engineering the full cell design, the porous CuO-NSs/RGO composite can be a promising anode material for high-performance LIBs.

\section{Experimental}

\section{Synthesis of graphene oxide}

Graphene oxide (GO) was prepared by a chemical oxidation process of graphite flakes (99.8\%, Alfa Aesar) using a modified Hummers' method. ${ }^{33}$ Briefly, graphite flakes (1 g) was mixed with concentrated $\mathrm{H}_{2} \mathrm{SO}_{4}(25 \mathrm{~mL})$ in a $250 \mathrm{~mL}$ of round-bottom flask under stirring with ice-water bath. $\mathrm{KMnO}_{4}$ powder $(3 \mathrm{~g})$ was then slowly added into the mixture, and stirring was continued for $2 \mathrm{~h}$. The mixture was then kept at $35^{\circ} \mathrm{C}$ for $30 \mathrm{~min}$ of stirring. Next, ultra-pure water $(100 \mathrm{~mL})$ was slowly poured into the mixture with cold-water bath and then moved in oilbath pot and heated to $90{ }^{\circ} \mathrm{C}$ for 15 min under stirring. Afterwards, the mixture was poured into a $500 \mathrm{~mL}$ of beaker, $5 \mathrm{~mL}$ of $\mathrm{H}_{2} \mathrm{O}_{2}(30 \%)$ was then added into the mixture under stirring and a brilliant yellow solution was formed. The solution was centrifuged and washed with $\mathrm{HCl}$ aqueous $\left(1 \mathrm{~mol} \mathrm{~L}^{-1}\right)$ and ultra-pure water. After the washing process, the resultant GO suspension was obtained. The aqueous GO suspension was then freeze-dried using a lyophilizer. After lyophilization, lowdensity and loosely packed GO was obtained.

\section{Preparation of pristine $\mathrm{CuO}$ and porous $\mathrm{CuO}-\mathrm{NSs} / \mathrm{RGO}$ composite}

Pristine $\mathrm{CuO}$ was synthesized by a facile two-step thermal treatment procedure. First, the solution of $\mathrm{Cu}^{2+}$ ions in toluene was prepared by a modification of the procedure reported by Yang et al. ${ }^{34}$ Typically, $1 \mathrm{mM}$ of $\mathrm{Cu}\left(\mathrm{NO}_{3}\right)_{2} \cdot 3 \mathrm{H}_{2} \mathrm{O}$ was dissolved in ultra-pure water $(50 \mathrm{~mL})$ in a $300 \mathrm{~mL}$ of beaker, ethanol $(50 \mathrm{~mL})$ containing $1 \mathrm{~mL}$ of dodecylamine (DDA) was then poured into the $\mathrm{Cu}^{2+}$ aqueous solution. After stirring for $3 \mathrm{~min}$, toluene (50 $\mathrm{mL}$ ) was then added into the beaker, and stirring was continued for $1 \mathrm{~min}$. A few minutes later, the aqueous phase was complete color bleached, which indicates that $\mathrm{Cu}^{2+}$ ions were transferred from water to toluene. The upper layer of the solution of $\mathrm{Cu}^{2+}$ ions in toluene was transferred into another container, $1 \mathrm{~mL}$ of hydrazine hydrate was added. After stirring for $30 \mathrm{~min}$, the mixture was then placed in oven at $50{ }^{\circ} \mathrm{C}$ for $1 \mathrm{~h}$. The final precipitates were collected by centrifugation, washed with absolute alcohol, and dried at $50{ }^{\circ} \mathrm{C}$. The CuO-NSs were then obtained after thermally treated the final precipitates in a muffle furnace at $300{ }^{\circ} \mathrm{C}$ for $2 \mathrm{~h}$.

The synthesis of porous CuO-NSs/RGO composite is similar to the pristine $\mathrm{CuO}$. Typically, CTAB (0.795 g) was firstly dissolved into $50 \mathrm{~mL}$ of toluene. Then, $20 \mathrm{mg}$ of as-obtained lyophilized GO was added into the above solution, with ultrasonic treatment for $2 \mathrm{~h}$ to form a homogeneous GO suspension. The solution of $\mathrm{Cu}^{2+}$ ions in toluene was then poured into the GO suspension under stirring. Afterwards, hydrazine hydrate (1 $\mathrm{mL}$ ) was injected into the mixture, and stirring was maintained for $30 \mathrm{~min}$. The overall mixture was then placed in oven at $50{ }^{\circ} \mathrm{C}$ for $1 \mathrm{~h}$. The final precipitates were collected by centrifugation, washed with absolute alcohol, and dried at $50{ }^{\circ} \mathrm{C}$. The powder was ground in an agate mortar and then placed in a muffle furnace at $200{ }^{\circ} \mathrm{C}$ for $2 \mathrm{~h}$ to obtain the porous CuO-NSs/RGO composite.

RGO nanosheets were synthesized as the similar procedure as the CuO-NSs/RGO composite but in the absence of the solution of $\mathrm{Cu}^{2+}$ ions in toluene.

\section{Characterization}

Material characterization. The phase composition and morphologies of the as-prepared products were investigated using X-ray diffraction (XRD, Rigaku, Ultima IV), transmission electron microscopy (TEM, JEOL, JEM-2100), and field emission scanning electron microscopy (FE-SEM, JEOL, JSM-6330f). Fourier transform infrared spectroscopy (FTIR, Thermo Nicolet, AVATAR 370) was used to characterize the structure of the obtained products. Raman spectroscopy (Jobin Yvon, Labram010) was recorded in the range of $200-2000 \mathrm{~cm}^{-1}$ using laser excitation at $632 \mathrm{~nm}$. The pore size distribution and specific surface area dates were obtained from $\mathrm{N}_{2}$ sorption analysis conducted at $77 \mathrm{~K}$ on an analyzer (Micromeritics, ASAP 2020 HD). Pore size distribution dates were obtained from adsorption branch using $\mathrm{BJH}$ model, and specific surface area was calculated according to the BET method. The thermogravimetric analysis (TGA, PerkinElmer, Diamond TG/DTA) was operated in the temperature range of $30-800{ }^{\circ} \mathrm{C}$ (heating rate: $10{ }^{\circ} \mathrm{C} \min ^{-1}$ ) under air.

Electrochemical measurements. The electrochemical performances were examined by the use of CR 2025 coin-type cells assembled in the argon-filled glove box (the contents of $\mathrm{H}_{2} \mathrm{O}$ and $\mathrm{O}_{2}$ are below $\left.0.9 \mathrm{ppm}\right)$. A mixture containing the assynthesized active material (pristine $\mathrm{CuO}$, porous $\mathrm{CuO}-\mathrm{NSs}$ / RGO composite, or RGO nanosheets, 80 wt\%), conducting acetylene black (10 wt\%), and poly(vinyl difluoride) (PVDF, $10 \mathrm{wt} \%$ ) dissolved in $\mathrm{N}$-methyl-2-pyrrolidinone (NMP) was then coated onto $\mathrm{Cu}$ foil, and dried in a vacuum oven at $110{ }^{\circ} \mathrm{C}$ overnight. The $\mathrm{Cu}$ foil was then compacted, and cut into disks with $12 \mathrm{~mm}$ in diameter. Li foil was served as reference and counter electrode, microporous polypropylene was used as separator. $1 \mathrm{M} \mathrm{LiPF}_{6}$ in the $1: 1: 1$ (by volume) mixture of DMC/ $\mathrm{EMC} / \mathrm{EC}(\mathrm{DMC}=$ dimethyl carbonate, EMC = ethyl methyl carbonate, $\mathrm{EC}=$ ethylene carbonate) as the electrolyte. The 
recharge properties were investigated on a cell test system (Land CT2001A) in the voltage range from 0.005 to $3 \mathrm{~V}\left(v s\right.$. $\left.\mathrm{Li}^{-} \mathrm{Li}^{+}\right)$. CV and EIS were tested on an electrochemical working station (CHI 660B). EIS measurements were operated at a perturbation amplitude of $5 \mathrm{mV}$ in the frequency between 0.01 and $100 \mathrm{kHz}$. $\mathrm{CV}$ tests were measured at a scanning rate of $0.5 \mathrm{mV} \mathrm{s}^{-1}$ within a voltage between 0.005 and $3 \mathrm{~V}$.

Full cells were assembled by using porous CuO-NSs/RGO composite as anode and commercial LFP as cathode. The LFP cathodes were prepared by casting the slurry containing LFP powder (80 wt\%), conducting acetylene black (15 wt\%), and PVDF (5 wt\%) in NMP onto $\mathrm{Al}$ foil and dried in vacuum at $110^{\circ} \mathrm{C}$ overnight to evaporate the solvent. Before assembling full cells, the CuO-NSs/RGO anodes were pretreated to decrease the large initial irreversible capacity through an electrochemical activation process. The loading masses of CuO-NSs/RGO anode and LFP cathode were about 0.7 and $1.8 \mathrm{mg} \mathrm{cm}^{-2}$, respectively. The as-assembled full cell was tested between 0.8 and $3.8 \mathrm{~V}$, and the specific capacity of full cells was calculated based on cathode mass.

\section{Acknowledgements}

The authors are thankful for the financial support of the National Natural Science Foundation of China (No. 51238002, No. 51372080, and No. 51272075), and the Scientific Research Project of Education Department of Hunan Province (No. 16C0717). The authors also thank Dr Fan-Yan Zeng at Jiangxi Normal University for SEM and TEM analyses.

\section{Notes and references}

1 P. Poizot, S. Laruelle, S. Grugeon, L. Dupont and J. M. Tarascon, Nature, 2000, 407, 496-499.

2 M. V. Reddy, G. V. Subba Rao and B. V. R. Chowdari, Chem. Rev., 2013, 113, 5364-5457.

3 Z. Y. Wang, L. Zhou and X. W. (David) Lou, Adv. Mater., 2012, 24, 1903-1911.

4 A. K. Mondal, D. W. Su, S. Q. Chen, X. Q. Xie and G. X. Wang, ACS Appl. Mater. Interfaces, 2014, 6, 14827-14835.

5 F. X. Ma, H. Hu, H. B. Wu, C. Y. Xu, Z. C. Xu, L. Zhen and X. W. (David) Lou, Adv. Mater., 2015, 27, 4097-4101.

6 M. Zhang, M. D. Cao, Y. M. Fu, L. L. Xing, Q. Wang and X. Y. Xue, Mater. Lett., 2016, 185, 282-285.

7 M. D. Cao, M. Zhang, L. L. Xing, Q. Wang and X. Y. Xue, J. Alloys Compd., 2017, 694, 30-39.

8 L. L. Wang, H. X. Gong, C. H. Wang, D. K. Wang, K. B. Tang and Y. T. Qian, Nanoscale, 2012, 4, 6850-6855.

9 Z. C. Bai, Y. W. Zhang, Y. H. Zhang, C. L. Guo and B. Tang, Electrochim. Acta, 2015, 159, 29-34.

10 R. B. Wu, X. K. Qian, F. Yu, H. Liu, K. Zhou, J. Wei and Y. Z. Huang, J. Mater. Chem. A, 2013, 1, 11126-11129.

11 T. Chen, Y. Hu, B. R. Cheng, R. P. Chen, H. L. Lv, L. B. Ma, G. Y. Zhu, Y. R. Wang, C. Z. Yan, Z. X. Tie, Z. Jin and J. Liu, Nano Energy, 2016, 20, 305-314.

12 G. D. Park and Y. C. Kang, Chem.-Eur. J., 2015, 21, 91799184.
13 L. Shi, X. X. Fu, C. Y. Fan, S. Q. Yu, G. D. Qian and Z. Y. Wang, RSC Adv., 2015, 5, 85179-85186.

14 L. Q. Lu and Y. Wang, Electrochem. Commun., 2012, 14, 8285.

15 F. Janene, H. Dhaouadi, L. Arfaoui, N. Etteyeb and F. Touati, Ionics, 2016, 22, 1395-1403.

16 R. P. Zhang, J. Liu, H. G. Guo and X. L. Tong, Mater. Lett., 2015, 139, 55-58.

17 L. Shi, C. Y. Fan, C. X. Sun, Z. M. Ren, X. X. Fu, G. D. Qian and Z. Y. Wang, RSC Adv., 2015, 5, 28611-28618.

18 Z. C. Bai, Y. W. Zhang, Y. H. Zhang, C. L. Guo and B. Tang, Electrochim. Acta, 2015, 159, 29-34.

19 S. F. Zheng, J. S. Hu, L. S. Zhong, W. G. Song, L. J. Wan and Y. G. Guo, Chem. Mater., 2008, 20, 3617-3622.

20 J. Y. Xiang, J. P. Tu, J. Zhang, J. Zhong, D. Zhang and J. P. Cheng, Electrochem. Commun., 2010, 12, 1103-1107.

21 S. Ko, J. I. Lee, H. S. Yang, S. Park and U. Jeong, Adv. Mater., 2012, 24, 4451-4456.

22 Q. Wang, J. Zhao, W. F. Shan, X. B. Xia, L. L. Xing and X. Y. Xue, J. Alloys Compd., 2014, 590, 424-427.

23 Y. Liu, W. Wang, L. Gu, Y. W. Wang, Y. L. Ying, Y. Y. Mao, L. W. Sun and X. S. Peng, ACS Appl. Mater. Interfaces, 2013, 5, 9850-9855.

24 A. K. Rai, L. T. Anh, J. Gim, V. Mathew, J. W. Kang, B. J. Paul, N. K. Singh, J. J. Song and J. Kim, J. Power Sources, 2013, 244, 435-441.

25 F. Y. Zeng, Y. F. Kuang, G. Q. Liu, R. Liu, Z. Y. Huang, C. P. Fu and H. H. Zhou, Nanoscale, 2012, 4, 3997-4001.

26 G. Q. Tang, Z. G. Jiang, X. F. Li, H. B. Zhang, A. Dasari and Z. Z. Yu, Carbon, 2014, 77, 592-599.

27 G. Y. Li, B. H. He, M. Zhou, G. X. Wang, N. B. Zhou, W. Y. Xu, M. J. Jing and Z. H. Hou, Ionics, 2016, DOI: 10.1007/s11581016-1822-y.

28 B. C. Qiu, M. Y. Xing and J. L. Zhang, J. Am. Chem. Soc., 2014, 136, 5852-5855.

29 Z. H. Wen, Q. Wang, Q. Zhang and J. H. Li, Adv. Funct. Mater., 2007, 17, 2772-2778.

30 J. S. Zhou, L. L. Ma, H. H. Song, B. Wu and X. H. Chen, Electrochem. Commun., 2011, 13, 1357-1360.

31 T. Z. Yuan, Y. Z. Jiang, Y. Li, D. Zhang and M. Yan, Electrochim. Acta, 2014, 136, 27-32.

32 S. Mohapatra, S. V. Nair, D. Santhanagopalan and A. K. Rai, Electrochim. Acta, 2016, 206, 217-225.

33 W. S. Hummers and R. E. Offeman, J. Am. Chem. Soc., 1958, 80, 1339.

34 J. Yang, E. H. Sargent, S. O. Kelley and J. Y. Ying, Nat. Mater., 2009, 8, 683-689.

35 S. Stankovich, D. A. Dikin, R. D. Piner, K. A. Kohlhaas, A. Kleinhammes, Y. Y. Jia, Y. Wu, S. T. Nguyen and R. S. Ruoff, Carbon, 2007, 45, 1558-1565.

36 Z. Q. Jiang, Z. J. Jiang, X. N. Tian and L. J. Luo, Electrochim. Acta, 2014, 146, 455-463.

37 G. Q. Tang, Z. G. Jiang, X. F. Li, H. B. Zhang, A. Dasari and Z. Z. Yu, Carbon, 2014, 77, 592-599.

38 X. W. Liu, Y. Wu, Z. Z. Yang, F. S. Pan, X. W. Zhong, J. Q. Wang, L. Gu and Y. Yu, J. Power Sources, 2015, 293, 799-805. 
39 D. F. Qiu, B. Zhao, Z. X. Lin, L. Pu, L. J. Pan and Y. Shi, Mater. Lett., 2013, 105, 242-245.

40 J. Wang, B. Ding, X. D. Hao, Y. L. Xu, Y. Wang, L. F. Shen, H. Dou and X. G. Zhang, Carbon, 2016, 102, 255-261.

41 J. H. Lee, N. Park, B. G. Kim, D. S. Jung, K. Im, J. Hur and J. W. Choi, ACS Nano, 2013, 7, 9366-9374.

42 S. Laruelle, S. Grugeon, P. Poizot, M. Dolle, L. Dupont and J. M. Tarascon, J. Electrochem. Soc., 2002, 149, A627-A634.

43 S. D. Seo, D. H. Lee, J. C. Kim, G. H. Lee and D. W. Kim, Ceram. Int., 2013, 39, 1749-1755.

44 X. Y. Xue, P. Deng, S. Yuan, Y. X. Nie, B. He, L. L. Xing and Y. Zhang, Energy Environ. Sci., 2013, 6, 2615-2620.

45 A. Débart, L. Dupont, P. Poizot, J. B. Leriche and J. M. Tarascon, J. Electrochem. Soc., 2001, 148, A1266-A1274.
46 L. Martin, H. Martinez, D. Poinot, B. Pecquenard and F. L. Cras, J. Phys. Chem. C, 2013, 117, 4421-4430.

47 N. R. Srinivasan, S. Mitra and R. Bandyopadhyaya, Phys. Chem. Chem. Phys., 2014, 16, 6630-6640.

48 P. S. Veluri, A. Shaligram and S. Mitra, J. Power Sources, 2015, 293, 213-220.

49 W. X. Zhang, G. Ma, H. Y. Gu, Z. H. Yang and H. Cheng, J. Power Sources, 2015, 273, 561-565.

50 C. Wang, A. J. Appleby and F. E. Little, J. Electroanal. Chem., 2001, 497, 33-46.

51 C. K. Chan, R. Ruffo, S. S. Hong and Y. Cui, J. Power Sources, 2009, 189, 1132-1140.

52 R. H. Wang, C. H. Xu, J. Sun, L. Gao and H. L. Yao, ACS Appl. Mater. Interfaces, 2014, 6, 3427-3436. 